\title{
Novel Coronaviruses and Astroviruses in Bats*
}

\author{
Daniel K. W. Chu, J. S. Malik Peiris and Leo L. M. Poon ${ }^{* *}$ \\ ( State Key Laboratory of Emerging Infectious Diseases, Department of Microbiology, The University of Hong \\ Kong, Hong Kong SAR, China)
}

\begin{abstract}
Zoonotic transmissions of emerging pathogens from wildlife to human have shaped the history of mankind. These events have also highlighted our poor understanding of microorganisms circulated in wild animals. Coronaviruses and astroviruses, which can be found from a wide range of mammals, were recently detected in bats. Strikingly, these bat viruses are genetically highly diverse and these interesting findings might help to better understand the evolution and ecology of these viruses. The discoveries of these novel bats viruses not only suggested that bats are important hosts for these virus families, but also reiterated the role of bats as a reservoir of viruses that might pose a zoonotic threat to human health.
\end{abstract}

Key words: Coronavirus; Astroviruses; Emerging pathogens

Emerging and reemerging of zoonotic pathogens has posed an intense challenge to human even in the modern world. Even with the rapid advancement in technology and healthcare settings, we have never been closer to the victory in fighting against these infectious diseases. The emerging of highly pathogenic diseases in the past few decades, including AIDS, Ebola fever, avian influenza, neurological and respiratory diseases caused by Hendra and Nipah viruses, and SARS etc., caused enormous losses in economy and human lives. These zoonotic events have also demonstrated the vulnerability of the health of human and domestic animals.

Received: 2009-01-20, Accepted: 2009-02-10

* Foundation items: This work was supported by the National Institute of Allergy and Infectious Diseases (NIAID contract HHSN266200700005C) and European Research Project SARS-DTV (contract no: SP22-CT2004).

** Corresponding author.

Phone: +852-2819-9943, Fax: +852-2855-1241,

E-mail: 1lmpoon@hkucc.hku.hk
The expanding human population has shaped the emergence of infectious diseases in human or poultry by sustaining disease transmission with extremely high population density that did not exist anywhere in the world before $(6,28)$. Previous analysis has also shown that more than $70 \%$ of emerging or reemerging pathogens are known to be zoonotic (29), and many of these emerging infections are associated with wildlife (4). These observations suggested that human encroachment on wildlife habitats has caused the exposure of previously unknown pathogens in wild animals and resulted in increased transmissions between human, domestic animals and wildlife. The continuous expanding of animal farm industry together with the frequent travelling of human and livestock across different continents have also favored the transmission of these novel pathogens in immunological naïve human and animal populations worldwide. Researchers have also found that a significant portion of these newly emerged or reemerged pathogens are RNA viruses (29). 
An explanation for this is that RNA viruses might have much higher nucleotide mutation rates than those pathogens that have a DNA genome, thereby allowing the RNA viruses to better adapt to a new host (7).

Many emerging pathogens of human, domestic animals are assumed to be maintained in wildlife. However, many of these reservoirs for emerging viruses have never been identified. These perhaps reflect our poor understandings of animal pathogens circulating in the wildlife. Among different wild animals, bats might be one of the relatively well-studied wild animal populations in terms of zoonotic disease concerns. So far, over 60 viruses, including members from families of Rhabdoviridae, Orthomyxoviridae, Paramyxoviridae, Coronaviridae, Togaviridae, Flaviviridae, Bunyaviridae, Reoviridae, Arenaviridae, Herpesviridae and Picornaviridae, have been isolated or detected from different bat species (1, 20). Of these identified bat viruses, some are found to be the causative agents of disease in human (e.g. Ebola virus, Menangle virus, Rabies virus, Hendra virus and Nipah virus). It should be noted that bats represent the second largest order of mammals after rodents. The species richness of bats may harbor a great variety of viruses, thereby increasing the chances of generating viruses which can cross the species barrier. In addition, with its unique ability to fly and other special features (e.g. migration in some bat species), bats might have important roles for transmitting zoonotic pathogens to other animals and human. Here, we share our recent experiences on the discoveries of bat coronaviruses and astroviruses as examples to highlight this possibility.

\section{CORONAVIRUSES}

The discovery of a novel coronavirus as the causative agent for SARS in 2003 (22) surprised many virologists. Coronavirus, belongs to the family of
Coronaviridae, has a single-stranded positive sense RNA genome. Many coronaviruses are known to be found in mammals, including human, bovine, mice, swine, dogs and cats, and in some species of birds. Human coronaviruses were previously only known to be associated with mild respiratory or gastrointestinal diseases. However the unusual disease severity of SARS indicated that some coronavirus infections might be lethal. Sequence analysis on the SARS coronavirus showed that the virus is genetically distinct to all the other previously known coronaviruses in human or in other animals. Serological studies also revealed that there is no evidence of past infection of the virus in human. These findings suggested that SARS is a zoonotic event with an unknown animal reservoir. Surveillance for the animal source of the virus resulted in the detection of SARS-like coronavirus in civet cats and raccoon dogs in a wildlife market in China $(11,25)$. However, subsequence serological surveillance of these animals in farms and in other markets suggested that these wild animals might acquire the SARS-like virus from another animal source (26). In addition, experimental infection studies also indicated that civet cats might not the nature host of SARS coronavirus (30). Taken all these findings together, these civet cats and raccoon dogs in the wet market were unlikely to be the natural reservoir of the virus. Nonetheless, these wild animals in the wet markets clearly play as intermediate hosts in transmitting the viruses to humans.

The discovery of SARS-like coronavirus in civet cats trigged extensive surveillance of coronaviruses in wild animals. The discovery of the first coronavirus in bats was reported by us in 2005 (23). In this study, samples collected from 44 species of wild animals, including mammals, reptiles and birds, commonly found in Hong Kong were tested for coronavirus by RT-PCR assays. Positive PCR products were detected 
from both fecal and respiratory samples from three species of bent-winged bats (genus Miniopterus). These viruses could be detected in the same bat population throughout a year. In some sampling cohorts, the detection rate of the virus in Miniopterus species was as high as $63 \%$ in fecal samples. Sequence analysis on RNA dependent RNA polymerase (RdRp), helicase and spike genes of the virus indicated that it is a novel group 1 coronavirus. These interesting findings subsequently prompted extensive hunting for novel coronavirus in bats and resulted in discovering a great variety of novel groups 1 and 2 coronaviruses in Southeast China. Studies of these bat coronaviruses have revealed that the diversity of coronaviruses in nature is much greater than previously recognized. In addition to the detection of novel coronaviruses in Southeast China, other novel bat coronaviruses were also detected in North America (8) and Europe (10). Some of these bat viruses are phylogenetically distinct from all previously known coronaviruses. By contrast, some of these novel bat viruses form sister clades of other mammalian coronaviruses, suggesting that these virus might be closely related to the ancestors of coronaviruses found in other mammalian species (27). Population dynamics analysis showed that the populations of bats coronaviruses can be best explained by a constant population growth model, while coronaviruses in other mammals show epidemic-like increases in population (27). Based on these findings, it was proposed that bats are the nature hosts for coronaviruses.

Among these novel bats coronaviruses, a group of coronaviruses which are genetically closely related to SARS or SARS-like coronaviruses was discovered in Rhinolophus species (horseshoe bats) (16, 24). Serological studies indicated that there might be a high prevalence of SARS-like coronavirus infections $(28 \%-71 \%)$ in horseshoe bat populations (18). However, it should be noted that the sequence identity between SARS and bat SARS coronaviruses was shown to be only about $90 \%$, suggesting that these bat SARS viruses might not be the direct ancestors of human SARS coronavirus. In addition, the genetic relationships between SARS and bat SARS coronaviruses are further complicated by the fact that some of the bat SARS coronaviruses might be recombinants of the direct ancestor of human SARS coronavirus (15). Hence, further systematic surveillances of bat coronaviruses in horseshoe bats or other bat species are required to better understand the origin of SARS coronavirus.

\section{ASTROVIRUSES}

The discovery of a large diversity of astroviruses from different species of bats in Hong Kong was first reported in 2008 (2). Astrovirus, which belongs to the family of Astroviridae, consists of single-stranded positive sense RNA viruses with star-like appearance under electron microscope. This virus family has 2 genera, with the avastroviruses found in birds and the mamastroviruses found in mammals. Astroviruses have been identified from different mammals and birds including humans, bovine, swine, ovine, minks, dogs, cats, mice, chicken and turkeys. The mammalian viruses are often associated with gastroenteritis in most mammalian species, whereas the avian viruses are known to cause both intestinal and extraintestinal diseases (19). In human, astroviruses are the second or third most common viral agent found in children with diarrhea $(5,14)$, and these viruses can also cause significant diseases in elderly (17) and in immunocompromised patients (9). The prevalence of astroviruses in different species of apparently healthy Miniopterus bats roosting at a single cave in Hong Kong ranges from $36 \%$ to $100 \%$ throughout a year. This suggested that these astroviruses might persistently infect the same bat population. Interestingly, the genetic diversity of these viruses at a single habitat 
was found to be greater than the one for all the previously known mamastroviruses. More importantly, some bat astroviruses were found to be genetically closely related to human astroviruses, suggesting these bat and human viruses might share the same ancestor. A similar high prevalence of genetically diversified astroviruses has also been detected in bats captured from different part of China (unpublished data).

Based on a phylogenetic analysis on viral RdRp gene, astroviruses detected from two different host species (Miniopterus magnater and Miniopterus pusillus) were found to be clustered together, suggesting some of these bat astroviruses are more ready to cause zoonotic transmission. This observation is notably different from that observed from bat coronaviruses $(2,3)$. With these findings, it is tempting to speculate that some of these bat astroviruses might have a great zoonotic potential. Further investigations of bat astroviruses might provide new insights into the ecology and evolution of astroviruses.

\section{CONCLUSION}

The majority of previous studies of coronaviruses and astroviruses were limited to the detection of viruses in clinical and veterinary samples. In particular for astrovirus, genetic information was based on short fragments of viral DNA amplified by reverse transcription-PCR $(12,21)$. With the discovery of these novel coronaviruses and astroviruses in bats, unprecedented information about these two viral families can be generated. The availability of the genetic information enables us to elucidate the phylogenetic relationships and selection pressure and recombination events in these viruses. The information also helps the development of better detection methods for possible unknown coronaviruses and astroviruses which may cause diseases in livestock or in human. These results might also help to identify crucial viral features which might be useful for novel drug designs. More importantly, the discoveries of these novel bat viruses highlighted the role of bats as the major reservoirs of emerging viruses with zoonotic potential. As efficiency disease control must be directed at the reservoir (13), surveillance for unknown pathogens in wildlife is a step needed to be taken for our better preparedness for future zoonotic diseases.

\section{References}

1. Calisher C H, Childs J E, Field H E, et al. 2006. Bats: important reservoir hosts of emerging viruses. Clin Microbiol Rev, 19:531-545.

2. Chu D K, Peiris J S, Chen H, et al. 2008. Genomic characterizations of bat coronaviruses (1A, 1B and HKU8) and evidence for co-infections in Miniopterus bats. J Gen Virol, 89:1282-1287.

3. Chu D K, Poon L L, Chan K H, et al. 2006. Coronaviruses in bent-winged bats (Miniopterus spp.). J Gen Virol, $87: 2461-2466$.

4. Cleaveland S, Laurenson M K, Taylor L H. 2001. Diseases of humans and their domestic mammals: pathogen characteristics, host range and the risk of emergence. Philos Trans R Soc Lond B Biol Sci, 356:991-999.

5. Dalton R M, Roman E R, Negredo A A, et al. 2002. Astrovirus acute gastroenteritis among children in Madrid, Spain. Pediatr Infect Dis J, 21:1038-1041.

6. Daszak P, Cunningham A A, Hyatt A D. 2000. Emerging infectious diseases of wildlife--threats to biodiversity and human health. Science, 287:443-449.

7. Domingo E. 2000. Viruses at the edge of adaptation. Virology, 270:251-253.

8. Dominguez S R, O'Shea T J, Oko L M, et al. 2007. Detection of group 1 coronaviruses in bats in North America. Emerg Infect Dis, 13:1295-1300.

9. Gallimore C I, Taylor C, Gennery A R, et al. 2005. Use of a heminested reverse transcriptase PCR assay for detection of astrovirus in environmental swabs from an outbreak of gastroenteritis in a pediatric primary immunodeficiency unit. J Clin Microbiol, 43:3890-3894.

10. Gloza-Rausch F, Ipsen A, Seebens A, et al. 2008. Detection and prevalence patterns of group I 
coronaviruses in bats, northern Germany. Emerg Infect Dis, 14:626-631.

11. Guan Y, Zheng B J, He Y Q, et al. 2003. Isolation and characterization of viruses related to the SARS coronavirus from animals in southern China. Science, 302:276-278.

12. Guix S, Bosch A , Pinto R M. 2005. Human astrovirus diagnosis and typing: current and future prospects. Lett Appl Microbiol, 41:103-105.

13. Haydon D T, Cleaveland S, Taylor L H, et al. 2002. Identifying reservoirs of infection: a conceptual and practical challenge. Emerg Infect Dis, 8:1468-1473.

14. Herrmann J E, Taylor D N, Echeverria P, et al. 1991. Astroviruses as a cause of gastroenteritis in children. The New England J Med, 324:1757-1760.

15. Hon C C, Lam T Y, Shi Z L, et al. 2008. Evidence of the recombinant origin of a bat severe acute respiratory syndrome (SARS)-like coronavirus and its implications on the direct ancestor of SARS coronavirus. J Virol, 82:1819-1826.

16. Lau S K, Woo P C, Li K S, et al. 2005. Severe acute respiratory syndrome coronavirus-like virus in Chinese horseshoe bats. Proc Natl Acad Sci USA, 102:14040-14045.

17. Lewis D C, Lightfoot N F, Cubitt W D, et al. 1989. Outbreaks of astrovirus type 1 and rotavirus gastroenteritis in a geriatric in-patient population. J Hosp Infect, 14:9-14.

18. Li W, Shi Z, Yu M, et al. 2005. Bats are natural reservoirs of SARS-like coronaviruses. Science, 310:676-679

19. Mendez E, Arias C F. 2007. Fields Virology, 5th ed, vol. 1. Lippincott Williams \& Wilkins, Philadelphia, Pa.

20. Omatsu T, Watanabe S, Akashi H, et al. 2007. Biological characters of bats in relation to natural reservoir of emerging viruses. Comp Immunol Microbiol Infect Dis, 30:357-374

21. Pantin-Jackwood M J, Spackman E, Woolcock P R. 2006. Molecular characterization and typing of chicken and turkey astroviruses circulating in the United States: implications for diagnostics. Avian Dis, 50:397-404.

22. Peiris J S, Lai S T, Poon L L, et al. 2003. Coronavirus as a possible cause of severe acute respiratory syndrome. Lancet, 361:1319-1325.

23. Poon L L, Chu D K, Chan K H, et al. 2005. Identification of a novel coronavirus in bats. J Virol, 79:2001-2009.

24. Ren W, Li W, Yu M, et al. 2006. Full-length genome sequences of two SARS-like coronaviruses in horseshoe bats and genetic variation analysis. J Gen Virol, 87:3355-3359.

25. Song H D, Tu C C, Zhang G W, et al. 2005. Cross-host evolution of severe acute respiratory syndrome coronavirus in palm civet and human. Proc Natl Acad Sci USA 102:2430-2435.

26. Tu C, Crameri G, Kong X, et al. 2004. Antibodies to SARS coronavirus in civets. Emerg Infect Dis, 10:2244-2248.

27. Vijaykrishna D, Smith G J, Zhang J X, et al. 2007. Evolutionary insights into the ecology of coronaviruses. $\mathbf{J}$ Virol, 81:4012-4020.

28. Wolfe N D, Dunavan C P, Diamond J. 2007. Origins of major human infectious diseases. Nature, 447:279-283.

29. Woolhouse M E , Gowtage-Sequeria S. 2005. Host range and emerging and reemerging pathogens. Emerg Infect Dis, 11:1842-1847.

30. Wu D, Tu C, Xin C, et al. 2005. Civets are equally susceptible to experimental infection by two different severe acute respiratory syndrome coronavirus isolates. $\mathbf{J}$ Virol, 79:2620-2625. 